\title{
HISTORY OF INPUT MODELING
}

\author{
Russell Cheng \\ Mathematical Sciences \\ University of Southampton \\ Highfield \\ Southampton, SO17 1BJ, UK
}

\begin{abstract}
In stochastic simulation, input modeling refers to the process of identifying and selecting the probability distributions, called input models, from which are generated the random variates that are the source of the stochastic variation in the simulation model when it is run. This article reviews the history of the development and use of such models with the main focus on discrete-event simulation (DES).
\end{abstract}

\section{INTRODUCTION}

As stated in the abstract, this article reviews the history of the development and use of input models in stochastic simulation. The focus is on discrete event simulation (DES), an early seminal work for which is Fishman (1973), but, given the use of stochastic simulation in so many diverse areas, mention will be made of interesting input modeling in simulations that might not be strictly considered as discrete-event. While the review covers work on input modeling that has appeared in the literature as a whole, special emphasis has been placed on what has been published in the Winter Simulation Conference (WSC) Proceedings as a fitting tribute to WSC at its fiftieth anniversary.

WSC has provided an internationally leading forum within which important topics of simulation in its diverse forms are presented and discussed, with particular emphasis on systems that occur in management science and operations research. This encompasses problems spread widely in many organizational and financial areas including manufacturing, marketing, construction, business, transportation, communications, healthcare and other service industries, military contexts, banking and insurance.

\section{$1.1 \quad$ Aspects of Input Modeling}

In our review we shall survey how the following aspects have developed and changed in the last 50 years:

A1: Determining the probability distributions from which random samples are drawn in running a DES model

A2: Fitting such probability distributions to historical data

A3: Bayesian approaches to distribution fitting

B1: Assessing the goodness-of-fit of fitted distributions: mathematical statistics approach

B2: Assessing the goodness-of-fit of fitted distributions: bootstrap approach

C: Modeling random variate dependencies: multivariate processes and non-stationary processes

D1: Mathematical algorithms for generating of random variates from specific distributions

D2: Resampling random variates from historical data

D3: Data preparation without historical data

E: Advances in input data gathering: improved procedures; improved software

F1: Measures of input uncertainty 


\section{Cheng}

F2 Mathematical analysis of input uncertainty

F3 Bootstrap analysis of input uncertainty

F4 Input uncertainty involving dependent input models

F5 Input uncertainty in simulation optimization

F6 Input uncertainty in specific application areas

Input uncertainty, which appears six times in the list, refers to the uncertainty that occurs in the process of selecting and determining input models and the knock-on effect that this has when conducting ing experiments with the simulation model. The different forms of input uncertainty that can arise will be defined more fully in Section 3.1.

One aspect of our review needs to be noted straightaway. Use of statistical experimentation to solve difficult mathematical problems has always, at its heart, employed aspect D1: mathematical algorithms for sampling from given probability distributions. This dates back to the earliest historical times; consider for example Buffon's needle experiment to determine the value of $\pi$. This aspect is therefore an integral part of input modeling.

However, such is the importance of D1, it can be treated as being a topic in its own right. Though it is beyond the scope of this review to discuss D1, many WSC Proceedings articles, as well as in the literature as a whole, have been written on random variate generation. A good comprehensive reference is Devroye (1986).

\subsection{Growth of Input Modeling}

Right from the start, the Winter Simulation Conference has provided an excellent vantage point from which to take in what is topical and of current interest in the field of DES. We therefore first demonstrate how input modeling has grown in the past 50 years simply by counting the number of articles in each year that involve, with the exception of D1, one or more of the aspects listed above. This WSC count does not of course have the same weight as one stemming from a comprehensive literature review, but nevertheless it provides a good indicator of how things have developed.

Figure 1 shows the number of such articles that have appeared in the Proceedings of the WSC through the years 1968-2016. Our decision to include or exclude certain articles is somewhat subjective. However, as already stressed, one choice is very explicit. Articles on D1, algorithmic random variate generation are excluded; but articles on D2 concerning variate generation by resampling are not excluded. Over the period of our review, there was much initial interest in D1, but it had dwindled by the mid 1980's. Thus the inclusion of articles on variate generation in the count would have lessened the upward trend seen in the graph. However, as we shall see, there is a genuine increase in the number of articles appearing each year concerning some aspect of input modeling that is not simply explained as being part of a more general growth in the number of DES articles. The increase is due to new developments, three of which we briefly highlight immediately.

One is the aspect of input uncertainty, already mentioned in Subsection 1.1. Since the mid 1990's there has been increasing recognition its importance. Our inclusion of the topic as an aspect of input modeling means that the growing number of articles on this topic has been a contributory factor in the overall increase of input modeling articles in WSC proceedings since the mid 1990's, as shown in Figure 1. Aspects F1 through F6, which all concern input uncertainty are reviewed in Sections 3 and 5.

A second aspect that has grown over the years is the use of inputs that are correlated or are nonstationary. Aspect $\mathbf{C}$ relates to this and practical problems where this has been studied are reviewed in Section 6.

A third development of note is the increased use computer intensive resampling techniques and, with the advent of big data, of data-driven simulation, where simulation experimentation is carried out using real data as the input, so that there is no need to develop explicit input models as such. The emphasis is thus on the aspects D2, D3 and $\mathbf{E}$, so that if we view these as part of input modeling, as we shall do, this 


\section{Cheng}

also boosts the input modeling article count since the mid 1990's. We review work on data-driven simulation in Section 7.

The closely related bootstrap method features in our list, explicitly under B2 and F3, but also implicitly under other headings, and this has had a similar contributory effect on the graph in Figure 1. Bootstrapping is discussed in Section 4, but only briefly because of lack of space (despite this author's own fondness of the topic; see Cheng, 2017).

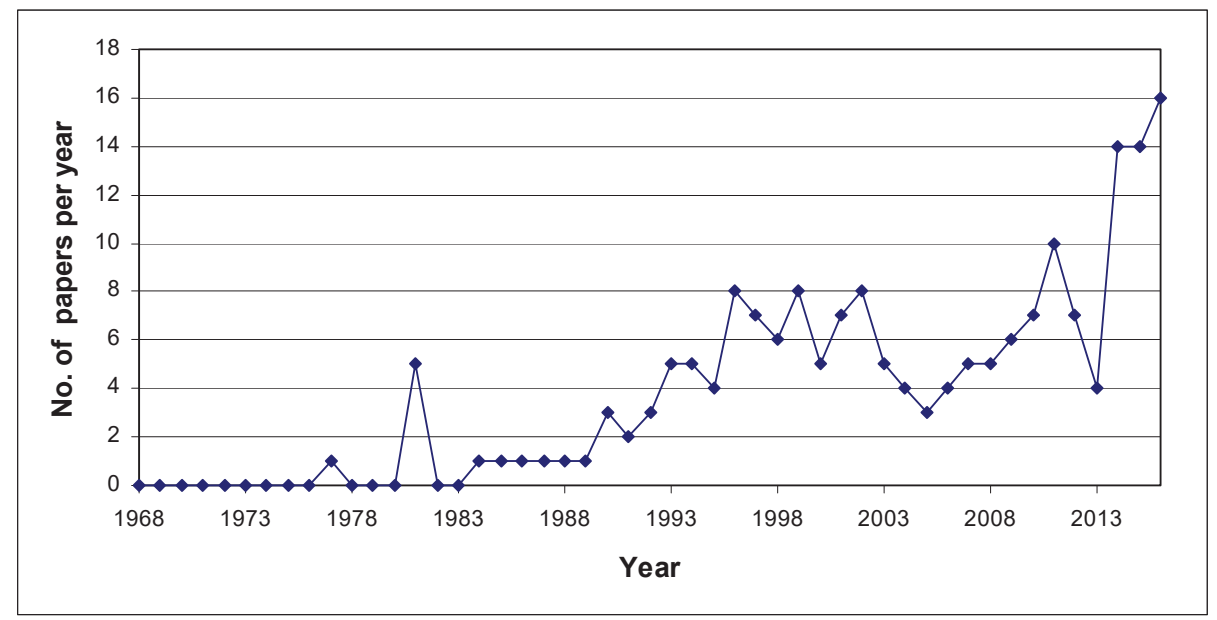

Figure 1: Number of WSC Proceedings articles each year involved with input modeling.

\subsection{Terminology}

At this point the reader may wonder why the term input modeling, in contrast to output analysis, was so little used in the early development of computer simulation as a subject. Particularly strange is that the term was not coined more rapidly in the period from the mid 1960's to the mid 1980's when interest in random variate generation methods was at its height. The reason seems to be one that is of historical interest.

The term input modeling seems most closely associated with DES rather than computer simulation in general. Performing computer-based statistical experiments to solve mathematical problems goes back centuries, an early example being the celebrated needle experiment discussed by Leclerc, later Comte de Buffon, in 1733. Since about 1944 (see, Hammersley and Handscomb, 1964), such experiments have been called Monte-Carlo methods, in which calculation of the quantity of interest in the system is considered to be a deterministic multiple integral, but which is calculated by a numerical quadrature involving probabilistic sampling. In his seminal book, Fishman (1996, Section 1.3) points this out, recognizing that DES is at heart a Monte Carlo method, but is an 'advanced topic' within this more general framework. For example if we use DES to evaluate a steady-state average of some quantity of interest in a queue, we are simply treating this as being a statistical expectation, calculated by a simulation run that amounts to a numerical quadrature of a massively high dimensional integral actually of the order of the number of individual variates sampled not just the number of input variable streams.

From the Monte Carlo viewpoint, the probability distributions involved are regarded as part-andparcel of the statistical experiment, and so are not treated as 'input distributions'. Thus in the early literature on DES, when it was still viewed as a Monte Carlo process, statistical distributions and the generation of random variates from these distributions were taken as being at the core of the experiment, and so were not regarded as being mere 'inputs'.

In our historical survey, covering the period that starts just after the mid 1960's, we are considering a body of work that on the one hand is influenced by the seminal monograph, Monte Carlo Methods, by 


\section{Cheng}

Hammersley and Handscomb (1964), in which the term input modeling is not used. On the other hand the body of work is influenced by The Art of Simulation by Tocher (1963), which is definitely a seminal book on DES, but where DES is still viewed as a statistical Monte Carlo experiment. So there is again no mention of input modeling. Both books describe in detail probability distributions useful in computer simulation and methods of variate generation from these distributions, without specially thinking of these aspects as being input modeling.

A final point concerning the term input modeling is that, though now more widely employed, it is still not used universally and seems unlikely ever will be. For example, in his two books on Monte Carlo Fishman $(1996,2006)$ does not use the term, and even in his book on DES, Fishman (2001) refers, and then only briefly, to input preparation and input process, though in all three references variate generation or its equivalent sampling from probability distributions is covered in detail.

Even what many regard as the standard reference on DES, Law (2015, Chapter 6), uses the term input probability distributions rather than input models.

We begin our review with a survey of the expository, mainly tutorial, articles that have been published in WSC Proceedings as this provides a convenient timeline to underpin our subsequent discussion. The rest of our article examines the aspects given in our list above more fully.

\section{WSC TUTORIAL PAPERS ON INPUT MODELING}

Early input modeling as applied in DES modeling, is slanted towards the Monte Carlo viewpoint where the objective is to determine specific probability distributions, usually of named form, like the lognormal, gamma, beta distributions, from which random samples will be drawn in running the simulation model. There are then three main aspects: A1: Selecting the probability distributions that might be used, A2: Fitting such probability distributions to existing data, and B1: Assessing the adequacy of the fitted distributions, typically using goodness-of-fit (GoF) tests.

Since 1986, WSC has included tutorials where these three aspects are the dominant theme, though other aspects of interest increasingly recognized as also being important are discussed as well. Foremost amongst tutorial contributors are Leemis $(1994,1995,1996,1997,1998,1999,2000,2001,2003,2004)$ and Law $(2011,2012,2013,2016)$. In the References, we have listed only selected articles by these and other authors as examples, so as not to over-burden the bibliography, as many of the articles cover essentially the same material, and there is easy online access to all the references for the interested reader. Thus in some citations with multiple dates listed, not all the dates may have a corresponding actual entry in the reference list.

Preceding these references Law and Vincent (1988) and Vincent and Law (1990) discuss the software package, originally called UniFit/UniFit II, now called ExpertFit for fitting many named distributions.

In an early paper aimed at practitioners Schmeiser (1977) was already surveying models that would give more flexibility than 'standard' distributions like the normal or lognormal. Kelton (1984) was the first paper advertised as a general tutorial on input data collection and analysis. Kelton discusses all three main aspects, namely distribution selection, fitting and assessing the quality of fit, and in addition also considers aspect D2, the use of sampling from existing data as an alternative to variate generation from a probability distribution, as well as aspect $\mathbf{C}$, modeling of multivariate and non-stationary processes. The first paper advertised as an introductory tutorial was given by Iman (1986). Though his discussion is quite general, Iman does consider aspects $\mathbf{D} 2$ and $\mathbf{C}$ as well. The three main aspects are also discussed in the tutorial by Johnson (1987), who already warns against use of overly rigid formal procedures for choosing input models. Kelton $(2007,2009)$ takes the issue of D2 further by considering the general question of how to represent uncertainty effectively. Sampling from an existing data has now extended into the datadriven method of simulation that is of considerable practical importance, and this will be discussed in Section 7.

Other workers have contributed WSC tutorials on input modeling: Cheng (1992, 1993, 1994) discussed variate generation aspects with the last paper focusing on use of bootstrapping in sampling 


\section{Cheng}

historical data. Wilson (1997) focused on modeling dependencies. Biller and Nelson (2002) highlighted important questions involved in input modeling. Lada et al. (2005) and Kuhl et al. (2006, 2008) consider the fitting of more advanced distributions like the Johnson, generalized beta, Bézier, and bivariate distributions. Biller and Gunes (2010) consider input models having non-standard marginal behavior or where dependencies are present as in a non-stationary Poisson process. Bézier systems are interesting as they are essentially non-parametric and so very flexible. However this does mean that their fitting is quite subjective, making it challenging to formally assess the quality of fit achieved.

Aspect $\mathbf{C}$ includes modeling of multivariate data using copulas as discussed for example by Nagaraj and Pasupathy (2015). A copula is a function of the components of a $d$-dimensional multivariate variable after it has been transformed so that it is distributed in a $d$-dimensional unit cube. The requirement is that each of the transformed components has a marginal distribution that is uniform $U(0,1)$, with the form of the copula retaining the interdependency between the components so that if the components are transformed back to their original form, they will recover the original dependence structure. The technicalities involved with using copulas make the topic somewhat advanced for an introductory tutorial. However use of copulas has become widespread in financial risk management, and since the global financial crisis of 2008 they are regularly used by financial regulatory bodies because of the insights and modeling capabilities that they provide in estimating multivariate extreme values. We shall return to this in Section 6.

A more accessible approach to the fitting of bivariate distributions, in particular bivariate Johnson distributions is considered by Wilson (1997).

As will be seen, since 1984 a baseline has been established in that there has been an introductory tutorial on input modeling almost every year, the basic content of which is little changed from year to year.

We now examine some of the input modeling aspects listed in Section 1.1 in more detail.

\section{UNCERTAINTY IN DES MODELS}

\subsection{Types of Input Uncertainty}

Our review is not intended to be in any way a tutorial in how to carry out input modeling. However, to appreciate more fully the kind of uncertainties that can arise it will be helpful to have a more detailed formulation of a simulation model as whole, and indeed of what constitutes a simulation. Song and Nelson (2013) capture the role of input models in a simulation model as follows: "Realizations from these input models combine with the simulation logic to produce output performance measures, and it is properties of these outputs ... that are used to infer what the real-world system performance would be".

We flesh out this description in slightly more mathematical detail by thinking of a DES model as representing a complicated stochastic process whose behavior is dependent on random variates drawn from $k$ input probability distributions (i.e. the input models) with cumulative distribution functions (CDFs)

$$
F_{j}\left(\cdot, \boldsymbol{\theta}_{j}\right), j=1,2, \ldots, k
$$

depending on parameters $\boldsymbol{\theta}_{j}, j=1,2, \ldots, k$, that are usually unknown. We assume that each run of the DES model requires samples

$$
\mathbf{x}_{j}=\left\{x_{j 1}, x_{j 2}, \ldots, x_{j m}\right\}, j=1,2, \ldots, k
$$

drawn respectively from each input model $j$. For simplicity of exposition we assume that each sample has the same size $m$, though this will not be so in general. Indeed the sample size will usually vary with input model and itself will be a random variable. 


\section{Cheng}

Also for simplicity, we assume that each sample $\mathbf{x}_{j}$ is a random sample where the sampled values $x_{j l}, l=1,2, \ldots, m$ are independently and identically distributed (IID) observations, but as already remarked, there are many important applications where samples have to be modelled that are correlated or non-stationary, and we shall consider this in more detail in Section 6.

We can view the behavior of the "simulation logic" alluded to by Song and Nelson (2013) as producing from the samples of Equation (2), in a simulation run, a univariate output response

$$
Y=Y(\mathbf{x}), \text { where } \mathbf{x}=\left(\mathbf{x}_{1}, \mathbf{x}_{2}, \ldots, \mathbf{x}_{k}\right),
$$

whose expected value $\mathrm{E}[Y(\mathbf{x})]$ is the performance measure of interest. If we use the output $Y$ from a single run as an estimator of $\mathrm{E}[Y(\mathbf{x})]$, then its variance $\operatorname{Var}(Y)$ is a measure of its accuracy.

$\operatorname{Var}(Y)$ is not usually of direct interest in itself, but may be used in calculating confidence intervals. Estimation of $\operatorname{Var}(Y)$ requires replication of runs. Suppose we make $r$ replications giving the outputs

$$
Y^{(i)}=Y\left(\mathbf{x}^{(i)}\right), \text { where } \mathbf{x}^{(i)}=\left(\mathbf{x}_{1}^{(i)}, \mathbf{x}_{2}^{(i)}, \ldots, \mathbf{x}_{k}^{(i)}\right), i=1,2, \ldots, r
$$

with $Y^{(i)}$ the output in the $i$ th replication depending on the vector of input variates $\mathbf{x}_{j}^{(i)}, j=1,2, \ldots, k$, each of which has the form $\mathbf{x}_{j}$ as in Equation (3).

$\operatorname{Var}(Y)$, the variability of the output observations, $Y^{(i)}, i=1,2, \ldots, r$, can be estimated using the usual sample variance $s^{2}$, where

$$
s^{2}=\sum_{i=1}^{r}\left(Y^{(i)}-\bar{Y}\right)^{2} /(r-1), \bar{Y}=\sum_{i=1}^{r} Y^{(i)} / r
$$

An example is the $\mathrm{M} / \mathrm{M} / 1$ queueing system where we suppose that the expected waiting time of a customer, $\mathrm{E}(W)$, is the performance measure of interest. We have exponential probability distributions, $\operatorname{Exp}(\lambda)$ and $\operatorname{Exp}(\mu)$ for the inter-arrival and service times of customers, so that $k=2$, with $\theta_{1}=\lambda$ and $\theta_{2}=\mu$ the two parameters of the system. These distributions are sampled to get the actual arrival and service pattern of the customers. In a simulation run of the model we might simulate the processing of $m$ customers with the average waiting time of the $m$ customers, $Y=\left(W_{1}+W_{2}+\ldots+W_{m}\right) / m$, estimating $\mathrm{E}(Y)=$ $\mathrm{E}(W)$.

Uncertainty arises in simulations in a number of different ways. We adopt the following terminology. Simulation Model Uncertainty: This is the uncertainty about the form of the simulation model itself, including, in particular, uncertainty about the precise form the 'simulation logic' referred to by Song and Nelson (2013). In our queue example, there may be uncertainty as to the queue discipline that should be simulated, for instance whether a queue capacity constraint should be imposed or not. We shall not discuss simulation model uncertainty very much as it is mainly an issue of the choice concerning the actual system to be simulated.

Simulation Uncertainty. This is the random variation that is explicitly simulated in a DES experiment by sampling from the input distributions. In our $\mathrm{M} / \mathrm{M} / 1$ queue example, when making a run of the queue, the random inter-arrival times and service times generated by the model may all affect the final output value $Y$. This form of uncertainty is an inherent part of experimentation with simulation models, and much of the early work on output analysis is targeted at estimating the variability in simulation output arising from it. The research on variance reduction methods is aimed at reducing this output variability.

Input Uncertainty: This is the uncertainty in the choice of the input distributions $F_{j}\left(\cdot, \theta_{j}\right)$ of Equation (1). It is convenient to think of this uncertainty as taking two forms, though as we shall show in a moment, the two forms can be treated in a unified way mathematically. 


\section{Cheng}

Parameter Uncertainty: This is the uncertainty where a parametric input model $F_{j}\left(\cdot, \theta_{j}\right)$ in Equation (1) is of known functional form but whose parameter values are unknown so that they have be estimated. For instance, in our $\mathrm{M} / \mathrm{M} / 1$ example, the inter-arrival time parameter $\lambda$ and the service time parameter $\mu$ can be estimated from samples of observed inter-arrival times and service times of actual customers. But the estimates $\hat{\lambda}$ and $\hat{\mu}$ are then random variables and will therefore have a statistical variation in their own right that affects the sample $\mathbf{x}_{j}$ of Equation (2), so contributing to the overall statistical variation of the output $Y(\mathbf{x})$.

Input Model Uncertainty: This is the uncertainty that arises because there may be some doubt as to which parametric model should be selected. For example should a gamma or a Weibull distribution be used? It is not absolutely necessary to think of parameter and input model uncertainty as being distinct, as different models can be combined into a single mixture model. For example if we are unsure as to whether $f(x, \theta)$ or $g(x, \varphi)$ is the more appropriate form for a particular input model we could use the mixture density $h(x, \theta, \varphi, w)=w f(x, \theta)+(1-w) g(x, \varphi)$ where $w$, with $0 \leq w \leq 1$, is an additional weight parameter also to be estimated. Viewed in this way only parameter uncertainty is involved.

Input uncertainty has been recognised formally, certainly by the mid-90s, and implicitly well before this time. Barton (2012) in a tutorial in WSC'12, mentions that Lee Schruben introduced the concept in discussion at WSC'92. Song et al. (2014) is an advanced tutorial paper on the topic. We give additional references in the following two subsections.

It should be noted that in the above discussion we have focused on input uncertainty arising in the case of parametric input models, whether it arises in the form of the parametric model to be selected or in the values of the parameters themselves. However input uncertainty can arise in a nonparametric setting where input models are simply thought of as being the underlying probability distributions that give rise to real data samples. Viewed in this way parametric models are then not absolutely needed, with simulation uncertainty, when running a model, obtained simply by bootstrapping as suggested by Barton and Schruben (1993). In fact if there is sufficient real data available then this can be directly sampled, treating the input models as being in effect completely known. This is what we discuss in the next subsection. This approach is the basis of data-driven models, which are reviewed in Section 7.

\subsection{Input Models Completely Known}

A very important case is the situation just mentioned at the end of the last subsection where we can sample from real processes to generate input streams. We then have an endless supply of variates drawn from existing real input processes which are therefore in effect known input models. For instance, in the single server queue example, we may be able use a dataset composed of observed service times of a real system. If the dataset is large enough we can generate service times when simulating the DES model, by drawing values without replacement from this dataset. However the dataset has to be large enough for all the service times, taking all $n$ simulation runs together as a whole, to be drawn without replacement. As will be reviewed later the growing area of data-driven simulation is one where input models are treated as being completely known.

This parameter free method of sampling from real data is statistically equivalent to having parametric input models whose true parameters values are effectively known, so that random variates can be drawn from this precise distribution. Thus $\operatorname{Var}(Y)$ is due to simulation uncertainty only, with no parameter uncertainty.

\subsection{Parametric Input Models}

We now consider the case where parametric input models are to be used.

There is one immediate warning. Use of the parametric input model approach does raise the possibility that the chosen models may not be suitable. Shanker and Kelton (1991) discuss the relative merits in simulation experiments of sampling input variates from an EDF corresponding to real data 


\section{Cheng}

compared with sampling from fitted parametric distributions, warning of the possibility of using a parametric input model whose distributional form is meant to correspond to that of existing real data, when it does not actually match. Goodness-of-fit testing using a sufficiently large data sample will prevent use of an input model that is seriously incorrect - a complication that is avoided using the parameter-free approach.

Suppose that we wish to use parametric input models with CDFs of the form (1), where the parameters $\theta_{j}, j=1,2, \ldots, k$, are unknown, but in contrast to Section 3.2, there is only a limited amount of real data for estimating them. We write $\boldsymbol{\theta}=\left(\boldsymbol{\theta}_{1}, \boldsymbol{\theta}_{2}, \ldots, \boldsymbol{\theta}_{k}\right)$. Parameter uncertainty is then present as well as simulation uncertainty.

The joint effect of simulation and parameter uncertainty on the output of interest was examined by Cheng and Holland (1995). They assume that each parameter $\theta_{j}$ is estimated from a real data random sample of the form $\mathbf{x}_{j}$ as given in Equation (2), only of sample size $n$ that is different from $m$, using the method of maximum likelihood (ML) yielding the overall ML estimator $\hat{\boldsymbol{\theta}}=\left(\hat{\boldsymbol{\theta}}_{1}, \hat{\boldsymbol{\theta}}_{2}, \ldots, \hat{\boldsymbol{\theta}}_{k}\right)$. For example, in the $\mathrm{M} / \mathrm{M} / 1$ example, suppose we have a sample of real inter-arrival times and a sample of real service times, both of size $n$, drawn respectively from the exponential distributions $\operatorname{Exp}(\lambda)$ and $\operatorname{Exp}(\mu)$. Then we can calculate ML estimators $\hat{\boldsymbol{\theta}}=\left(\hat{\theta}_{1}, \hat{\theta}_{2}\right)^{T}=(\hat{\lambda}, \hat{\mu})^{T}$.

Cheng and Holland (1995) showed that asymptotically.

$$
\operatorname{Var}[Y(\boldsymbol{\theta})]=r^{-1} \sigma^{2}\left(\boldsymbol{\theta}^{0}\right)+\mathbf{g}\left(\boldsymbol{\theta}^{0}\right)^{T} \operatorname{Var}(\hat{\boldsymbol{\theta}}) g\left(\boldsymbol{\theta}^{0}\right)
$$

where $\boldsymbol{\theta}^{0}$ is the true parameter value, $\sigma^{2}\left(\boldsymbol{\theta}^{0}\right)$ is the variance of the simulation uncertainty in one run, $\mathbf{g}(\boldsymbol{\theta})=\partial E[Y(\boldsymbol{\theta})] / \partial \boldsymbol{\theta}$, and $\operatorname{Var}(\hat{\boldsymbol{\theta}})$ is the variance-covariance matrix of $\hat{\boldsymbol{\theta}}$. The second term on the right is the parameter uncertainty due to having to estimate $\boldsymbol{\theta}$ by ML. This quantity is $\mathrm{O}_{\mathrm{p}}\left(n^{-1}\right)$. This shows simulation and parameter uncertainties are asymptotically separately and independently controllable. The simulation uncertainty is easily estimated and is simply controlled by increasing $r$ the number of runs. Likewise the parameter uncertainty is easily controllable by increasing $n$, the size of the data samples used to estimate $\boldsymbol{\theta}$.

Cheng and Holland $(1997,1998)$ discuss two methods for estimating the size of the parameter uncertainty. However this requires estimating $\mathbf{g}(\boldsymbol{\theta})$, which becomes increasingly expensive to do as the number of input models, $k$, increases. Freimer and Schruben (2002) discuss how much data to collect in order to identify important unknown parameters using designed experiments either with an analysis-ofvariance fixed effects model, or a random effects model. Ankenman and Nelson (2012) discuss a quick way to account for input uncertainty that was improved by Song and Nelson (2013). Lin et al. (2015) and Song and Nelson (2015) describe a way to estimate the gradient vector from just a single simulation run using a multiple regression approach, which has been implemented in Simio simulation software. Calculating confidence intervals that take into account parameter uncertainty has been studied by Cheng and Holland (2004) and Barton et al. (2013). Dependent input models have been studied by Xie et al. (2014a). Corlu and Biller (2015) consider subset selection under input uncertainty. Multivariate input uncertainty has been considered by Xie et al. (2016).

\section{SELECTION OF INPUT MODELS AND BOOTSTRAPPING}

Parametric input models are often selected on the basis of how well they fit a given sample of real data. Goodness-of-fit tests provide a formal way of comparing the fits of different fitted models. Law (2015) gives a detailed review of such goodness-of-fit tests.

Lack-of-fit is indicated by the $p$-value of a goodness-of-fit (GoF) test statistic. A small $p$-value is a strong indication of an unsatisfactory fit. However ranking the fits of different distribution using only the 


\section{Cheng}

$p$-value is commonly regarded as inadvisable. Kelton (1984) was already warning of over-reliance on $p$ values particularly when a large number of comparisons is being made.

Gupta and Parzen (2004) give a careful discussion of the selection of input models, pointing out that ranking the models according to the $p$-values of a goodness-of-fit test statistic can be misleading if used on its own. A GoF approach is over-formal, turning model selection into a 'hard' (in the sense of rigid) issue. Gupta and Parzen suggest model selection should be augmented by a softer but fuller approach involving for example visual examination of quantile-quantile (QQ) plots, and quantile-quartile (QIQ) plots.

A point of note is that the well-established chi-squared goodness-of-fit test is actually not a very sensitive test of fit, as well as being somewhat subjective, and is popular simply because it is easy to apply. The most sensitive tests have been long recognized, see Stephens (1974) for example, to be those based on EDF statistics, like $A^{2}$, the Anderson-Darling statistic or $W^{2}$ the Cramér - von Mises statistic. The difficulty of using such statistics is that their null distributions are not theoretically tractable and vary with the distribution being fitted and depend also on whether parameters are estimated, so that simple formulas for their p-values are not available. However, Cheng (2006, 2015), shows that it is very straightforward and easy to calculate the $p$-values of goodness-of-fit tests like the $A^{2}$ or $W^{2}$ statistics using bootstrapping, so that there is no real reason for not using these statistics in preference to the chi-squared test statistic.

An assessment of accuracy aspects of bootstrapping is discussed in detail by Barton and Schruben (1993) illustrated with a numerical example.

Bootstrap methods actually provide interesting alternative methods for estimating both simulation and input uncertainty. However lack of space prevents further discussion here. For the reader interested in the use of bootstrap methods in assessing uncertainty, a concise and clear account is given by Hjorth (1994). Chernick (2008) provides a comprehensive and very practical reference to bootstrapping methods useful for input modeling.

\section{BAYESIAN APPROACH TO INPUT UNCERTAINTY}

\subsection{Bayesian Formulations}

A Bayesian approach was originally considered by Chick (1997). This has been by extended by Xie et al. (2014b) who give a theoretical analysis and an empirical study of the basic parameter uncertainty problem and its effect on output using a fully Bayesian framework.

Zouaoui and Wilson (2003) use a Bayesian approach that also allows simulation and parameter uncertainties to be estimated. Zouaoui and Wilson $(2001,2004)$ extend this approach using Bayesian model averaging (BMA) to include estimation of input model uncertainty as well; see also Chick (2001).

A Bayesian formulation of input uncertainty requires a prior distribution to be assumed for the different forms that each input model may take and prior distributions for the parameters defining each form of each model. The posterior distributions of all the forms of each of the models and their defining parameters are estimated using the Markov chain Monte Carlo (MCMC) method. Zouaoui and Wilson (2001, 2004) develop a replication allocation that optimally allocates simulation runs to input models so as to minimize the variance of the estimated posterior mean response subject to a budget constraint on the total amount of simulated experimentation or computer time that is available.

When considering input model uncertainty, one might wish to evaluate input models that are of different dimensions. This occurs in fitting a finite mixture model where the number of components is unknown. Richardson and Green (1997) consider this problem and show that fitting by a Bayesian MCMC method is still possible, but this has to be done in a special way if the number of mixture components is unknown and also has to be estimated. They propose a reversible jump (RJMCMC) method which allows statistically valid jumps between models of different dimension when running the Markov chain. Using RJMCMC, the resulting posterior distributions of the fitted model can be quite 


\section{Cheng}

difficult to interpret. Cheng and Currie (2003) discuss an alternative Bayesian approach using an importance sampling method to generate the posterior distribution. The RJMCMC method and importance sampling methods are compared in Cheng (2017).

\subsection{Bayesian Approach Without Existing Data}

An interesting aspect of the Bayesian approach is that it can be applied extremely easily when there is no existing data with which to estimate unknown parameters of the input models. In the Bayesian approach we model the uncertainty in the correct parameter value $\boldsymbol{\theta}$ by thinking of it as being a random variable in its own right, so that it is treated as a quantity, which though fixed in a given run of the simulation model, will vary randomly between the different runs of the simulation experiment. The value used in each run is sampled from a (Bayesian) prior distribution which we denote by $\pi(\boldsymbol{\theta})$. This prior distribution of the parameter $\boldsymbol{\theta}$, has to be specified by the user, and will need to be chosen to reflect the user's uncertainty about the likely true value of $\boldsymbol{\theta}$.

The DES experiment involves making $n$ runs of the simulation model, where we simply make each run independently, in the $i$ th run first sampling just one $\boldsymbol{\theta}$ value, and then using this as the parameter value when generating random variates from the input distributions for that run.

The use of this Bayesian method is now widespread. L'Ecuyer in a private communication comments:

"I think stochastic models in general have become much more complicated than just i.i.d samples from one-dimensional distributions .... For example, think of choice models in marketing and transportation, travel time models, models in finance, biology, etc. The basic random variables that drive the models are often unobserved. There is a huge trend toward Bayesian statistics to be able to estimate these models. Such type of modeling is all over the place in machine learning now. They do that heavily at Google, Facebook, Amazon, etc."

The connection with machine-learning is a good one to make, as it emphasizes why the Bayesian approach is so attractive; namely that it (i) allows expert opinion to be incorporated in selecting $\pi(\boldsymbol{\theta})$ and also (ii) allows any random sample data, $\mathbf{x}$, whose distribution depends on $\boldsymbol{\theta}$, to be used together with the prior $\pi(\boldsymbol{\theta})$, to form a posterior distribution $\pi(\boldsymbol{\theta} \mid \mathbf{x})$, using the well-known Bayes formula, see Chick (1997) for example, which combines the prior and sample information on the parameter $\boldsymbol{\theta}$.

Thus the method can be used repeatedly thoughout a continuing investigation, allowing further data, or additional expert opinion, to be incorporated in updating and refining $\pi(\boldsymbol{\theta} \mid \mathbf{x})$, in a way that is logically very appealing.

\section{CORRELATED INPUTS}

Non-stationary, typically Poisson processes, and autoregressive process have been regularly studied in WSC Proceedings papers. We focus on fitting aspects rather than generation.

Kuhl et al. (1998) describe least squares estimation of nonhomogeneous Poisson processes. Cario and Nelson (1996) discuss processes which they term ARTA (a transformation from autoregressive to anything); Biller and Nelson (2002) giving an automated and statistically valid algorithm to fit such processes with marginal distributions from the Johnson translation system to stationary univariate timeseries data. Non-stationary Poisson processes are considered by Morgan et al. (2016).

Modeling the arrival and service processes of a queue has attracted special attention. Kuhl et al. (2003) describe an automatic procedure for estimating processes that exhibit long-term trends and nested periodic effects. Bause et al. (2009) consider Markovian arrival processes which are commonly used to describe correlated arrivals, where often ARMA/ARTA-based models are used. The authors compare experimentally the models according to their expressiveness and modeling capabilities for dependent sequences, finding that ARMA/ARTA-based models have advantages, though their fitting is more elaborate. Uhlig et al. (2016) review, from a practitioner's perspective, available tools to fit and model autoregressive input processes, reassuringly finding that tools relying on autoregressive models performed the best. 


\section{Cheng}

Call center arrival processes have been surveyed by Ibrahim et al. (2012). Oreshkin et al. (2016) review some key issues when modeling the time-dependent and stochastic arrival rate in service systems in general, and compare various input models. Avramidis et al. (2004), and Avramidis and L'Ecuyer (2005) discuss arrival models at telephone call centers in detail. An interesting issue is when the rate itself is not observed, but it still has to be modeled. Modeling of features for which there is little data, is an interesting area in data-driven simulations.

When there are different types of arrivals (different call types, or different types of customers, or different types of patients in clinics, or events in emergency systems, etc.), there is also dependence between arrival rates in the different arrival processes. This is discussed in Jaoua et al. (2013).

Ibrahim et al. (2016) consider examples of dependence in service times data; some unusual. For example, the service time distribution for a given call type often depends very strongly on the individual server, and is also time-dependent.

In discussing data dependency, the use of copulas deserves mention. Kurowicka and Cooke (2002) examine use of the Vine copula for representing high dimensional dependent distributions. Avramidis et al. (2009) discuss efficient correlation matching for fitting discrete multivariate distributions with arbitrary marginals and normal copula dependence. Channouf and L'Ecuyer (2009) examine fitting a normal copula for a multivariate distribution with both discrete and continuous marginals. Channouf and L'Ecuyer (2012) consider a normal copula model for the arrival process in call centers.

Copulas have been found to be especially useful in financial risk management where they are a powerful tool in modeling correlations in extreme values. The field is a rapidly expanding one. Some interesting recent advances are discussed by Embrechts et al. (2015) who investigate model uncertainty of regulatory risk measures highlighting the unsatisfactory nature of the commonly used VaR measure, Chavez-Demoulin et al. (2016) consider an extreme value approach for modeling operational risk losses, and Embrechts et al. (2016) examine tail-dependence compatibility. A very well cited introduction by Embrechts et al. (2017) is available online.

\section{DATA-DRIVEN DES}

In the present age of big data, data-driven simulation has come into its own, modeling systems that are complicated in the widest sense. In this kind of simulation, input models do not really appear explicitly, and even if recognised as such, have boundaries within the model that are not well defined. In effect the data itself is the model, to be sampled and interpreted in its own right. The emphasis is on dataacquisition, data-structuring, data-delving and data-interpretation with inference as the goal.

The field is now vast. In the space available, we can only give a flavour of it with a few examples, drawn mainly from WSC Proceedings papers.

Tannock et al. (2007) consider use of data-driven simulation to assess the performance of supply chains in aerospace manufacturing. In this the simulation model is constructed automatically using data from company IT systems. The paper describes such a supply-chain model builder, with an example provided from the civil aerospace sector. Though not a replacement for general purpose simulation tools the data-driven model does allow a variety of scenarios to be rapidly explored without a high level of simulation expertise.

Skoogh and Johansson (2008) discuss DES projects that rely heavily on high input data quality. The paper presents a structured methodology for handling such input data, including description of 13 activities and their internal connections. The methodology increases speed in input data management and, consequently, in carrying out entire DES projects. The improvement is expected to be larger in companies with low or medium experience in DES.

Bengtsson et al. (2009) describe a methodology for input data management (IDM) in DES projects. The approach provides a methodology to identify and collect data, then uses IDM software to extract and 


\section{Cheng}

process the data. The IDM software structures and presents the data in Core Manufacturing Simulation Data (CMSD) format, which is aimed to be a standard data format for any DES software. The IDM methodology was previously developed and tested by Chalmers University of Technology in a case study in the automotive industry. This paper presents a second test implementation in a project at the National Institute of Standards and Technology (NIST) in collaboration with an aerospace industry partner.

Konrad and Lawley (2009) consider health care management. A variety of simulation models of hospitals exist; however, few reflect resource sharing across multiple departments or capture the heterogeneity of patient mix. The paper highlights how flow path approaches to studying hospital operations have been hindered by lack of a comprehensive data source, describing how electronic communication exchanges between hospital departments can be used to create an input model for hospital simulations.

Huang et al. (2011) propose a data-driven approach for automatic model generation using pre-built and validated model components in which models can be rapidly generated by automatically selecting, structuring and configuring the model components.

Henclewood et al. (2012) discuss an interesting application of real-time data-driven simulation where calibration tools are shown to be needed in measuring traffic congestion under different conditions.

Rabe and Scheidler (2014) discuss complex large scale input data management and precise model design in supply chain management showing how they can be complemented. They discuss how Knowledge Discovery can be applied, as a preprocessing step for simulation scenario setups, in order to provide benefits for the level of accuracy in the resulting simulation models.

Kotiadis (2016) gives a useful review of simulations involving data-driven input models in large-scale practical applications.

Barlas and Heavey (2016) point out that the input data process is usually $10-40 \%$ of the total time of a DES project. They describe a tool implemented in OS software, called the Knowledge Extraction (KE) tool, that can extract raw data from a company's data sources, and transform this data to simulation input, giving three case studies where the tool has been implemented.

The paper by Matus et al. (2016) illustrates the complications involved in fitting what might be considered to be the input model. The authors calibrate various Marshall-Olkin (MO) copula models so as to attain given failures that arise simultaneously in groups of links in a telecommunication network. Simulating these models shows that considering the simultaneous failures of small and connected subsets of links is the key to obtaining a good approximation of reliability, confirming what is suggested by the telecommunication literature.

\section{PERSONAL EXPERIENCES}

Authors of the WSC'17 History Track have been encouraged to include personal experiences of their own, to give a more human dimension to our professional work. The personal experiences that are described in this section are probably best regarded as involving stochastic modeling, rather than purely input modeling in DES simulation. However they all involve parameter estimation and indeed variate generation, so hopefully can charitably be regarded as falling within the purview of this article.

I shall begin with a story, in which I do happen to have an incidental role. However it is really one that Richard (Dick) Nance, the 2006 winner of the INFORMS Simulation Society's Lifetime Professional Achievement Award, has kindly shared with me. It amusingly illustrates how the resolve displayed by the mathematically able in focusing on high-powered research often has to be tempered by lower level solution methods, such as simulation, when tackling actual practical problems.

As part of his doctoral work at Purdue, Dick Nance took courses in statistics, including a challenging one given by Marcel Neuts on stochastic processes. At the time, 1966, Neuts was publishing papers on queueing theory very regularly in Operations Research. However Neuts formed a more personal relationship with Dick as he was very interested in learning about Dick's knowledge in computing and programming capability. However Neuts had a very strong disdain for simulation (Dick thinks probably 


\section{Cheng}

because Neuts feared that the limitations of analytic techniques and solutions in terms of transforms in which he was expert could become passé). He therefore enjoyed giving Dick a hard time about his research interests in simulation, once commenting to a seminar audience: “... or you could do as Mr. Nance prefers and use simulation thus abrogating your mathematical birthright." Dick took the "slur" in jest, and retained a friendly relationship. Some years later Dick was shocked to see Neuts' name on a paper presented at WSC, Neuts and Pagano (1981), thinking: 'how could Neuts possibly venture into this den of mathematical iniquity?'

I can add a personal flourish to Dick's story. At that time, I had a colleague, Professor Jeff Griffiths, like Neuts a queueing specialist, renowned both nationally and internationally for his work on high profile queues like the Suez Canal. Jeff, like Neuts, preferred analytic techniques in solving queueing problems, and had indeed discussed with Neuts problems of mutual interest. However Jeff found that Neuts' mathematical methods could be quite unstable numerically, and so often resorted to simulation. This was in the mid 1970's, and I still clearly remember the coffee break when Jeff had remarked to me that there did not seem to be any good simple gamma variate generators. I checked and found this was indeed the case and it led to my developing my GB gamma variate generator proposed in Cheng (1977). I guess Neuts must have had the same difficulty and resorted to simulation, of which his WSC'81 Proceedings with Pagano was a consequence. WSC'81 was the first WSC that I attended, and I did meet Neuts and Miriam Pagano. Their paper was on the generation of random variates from phase-type distributions, and rather pleasingly for me they had used my GB generator in their paper.

My second story features David Kendall, also an eminent queueing theorist. He was Director of the Statistical Laboratory at Cambridge University, when I studied there. Like Dick with Neuts, I knew Kendall personally having attended seminars by him. It was he who proposed the $M / M / 1$ notation used in classifying queues, and as a lowly graduate student I held him in awe, especially when I once bumped into him in a corridor, when he was conversing fluently in Romanian with a visitor. After I left Cambridge I followed his work. Kendall's work is noted for being theoretically insightful and with results always elegantly derived using powerful mathematics. But in 1974, he encountered hubris in his paper, Kendall (1974), on pole-seeking Brownian motion, applying this to the movements of migratory birds. Kendall was a keen ornithologist. In the paper was the declaration 'For mathematical purposes, ...., the von Mises distribution is very attractive, but it has the great disadvantage that it is virtually impossible to simulate!'

The publication was a prestigious Royal Statistical Society 'read' discussion paper, meaning one that is presented at a general meeting of the Society, with preprints previously circulated so that Fellows of the Society can comment at length personally on the paper at the meeting (but timed in those days by an eggtimer!), with the Discussion published subsequently with the paper. Kendall's ignorance of the acceptance-rejection method for generating random variates was commented on by several discussants. Kendall apologized in writing, graciously remarking: 'I fully acknowledge my ignorance, commented on by several speakers, of the rejection technique for simulating random variables with a given density. I shall know better next time.'

Though Kendall claims 'the paper is not a mathematical one', it is only fair to end by saying that the paper is actually a superb and masterly tour de force of probabilistic analysis, one that Marcel Neuts would certainly have approved of.

I turn now to two personal experiences that I remember well. Both have a NASA angle.

The first involved fitting Weibull distributions to model the strength of brittle materials and for me the work brought home and illustrated the importance of good methodology.

Neil Cooper and Jim Margetson, material scientists in the United Kingdom Ministry of Defence (MoD), were studying components in rocket motors made from ceramic and graphite; see for example Cooper et al. (1986). They had samples of elementary rectangular shaped bars of such material whose breaking strength they measured in simple laboratory experiments. They used a probabilistic approach by fitting a Weibull model to the samples of observed highly variable brittle strengths. This would show how 


\section{Cheng}

the breaking stress depended on the Weibull parameters. A quite complicated finite element model was then used to predict the strength of actual rocket components, whose shape was much more complicated.

When I and two departmental colleagues, Brian Evans and Janet Williams, were first involved, the MoD were using graphical methods to estimate the Weibull parameters. Their methods were very thoughtfully devised, but rather elementary. We received a number of contracts to work on this problem over a number of years in the late 1980's, and we developed a maximum likelihood method for carrying out the full analysis which produced confidence bands for the CDF of the breaking stress of actual components. This work has been reported in Cheng et al. (1988).

Towards the end of these contracts, I remember that Jim Margetson, after a long meeting, threw his pencil down on the table which was covered with papers and notes, and leant back with a sigh. I was worried as he appeared displeased. But instead he said 'I wish I had known about maximum likelihood years ago!'

There is a rider. NASA at the time had put in significant resources studying the same problem. I had actually been somewhat disappointed by our results as there was a clear bias. However we were able to compare them with NASA's results which turned out to be similarly biased. Looking at the model now with the benefit of 30 additional years of experience, I think I would want to try a shifted Weibull distribution!

Jim left the MoD not long afterwards, developed a commercial version of the method, with very nice graphical displays of the stresses in the finite element components, which he then successfully marketed to the Canadian National Defence Department.

My second story involves teaching. It brought home to me very forcefully that though our teaching of probability might sometimes appear academic, nevertheless its importance can be perilous to ignore.

In 1985-6. I was on sabbatical leave visiting the Curriculum in Operations Research at UNC, Chapel Hill, at the kind invitation of George Fishman, its then Chair. While there I taught a course, ORSA 181, whose precise title I now forget, but which was essentially Stochastic Modeling. This included study of the Poisson distribution. I found the usual textbook illustrations of its 'use' rather mundane, like estimating 'the number of typographical errors on a printed page'.

At the time, the space shuttle program was in full swing, and much was being made of the shuttle's reliability, with NASA promoting its possible commercial use. Just before discussing the Poisson distribution in lectures, I happened to come across an article in the local Chapel Hill newspaper describing the construction of the shuttle in engineering terms, which appeared to confirm its overall reliability. The article also mentioned that about 200 more launches were planned in the overall shuttle program. Though the article did not provide a probabilistic risk analysis, enough information was given for an educated estimate to be made assuming a Poisson failure model. With the figures that were given, the estimate of the probability of no serious launch failure over the planned entire program of 200 launches, was no better than $50 \%$.

I used it as an example in class, asking whether the probability of launch failure was small enough to warrant promotion of the program as being safe for commercial exploitation. This was in the third week of January 1986.

I little thought that in the very next week, the launch of Challenger would go so disastrously wrong, carrying with it the dreadful fate of the entire crew, and in particular of the teacher Christa McAuliffe, only on board as NASA tried to demonstrate that the space shuttle was safe enough for use by 'normal' people.

I can only lamely hope that, of all the students I have taught, at least that class appreciated the value of understanding the Poisson distribution.

\section{CONCLUSIONS}

When I started writing this article I wondered if there would be much to say about input modeling. However if a flexible viewpoint is taken of what constitutes an input model, then the area is an amazingly 


\section{Cheng}

rich and varied one, with many interesting aspects, both theoretical and practical, worthy of additional study.

\section{REFERENCES}

Ankenman, B. E. and B. L. Nelson. 2012. “A Quick Assessment of Input Uncertainty”. In Proceedings of the 2012 Winter Simulation Conference, edited by C. Laroque, J. Himmelspach, R. Pasupathy, O. Rose, and A. M. Uhrmacher, 241-250. Piscataway, New Jersey: Institute of Electrical and Electronics Engineers, Inc.

Avramidis, A. N., N. Channouf, and P. L'Ecuyer. 2009. "Efficient Correlation Matching for Fitting Discrete Multivariate Distributions with Arbitrary Marginals and Normal Copula Dependence". INFORMS Journal on Computing, 21, 1, 88-106.

Avramidis, A. N., N. Deslauriers, and P. L'Ecuyer. 2004. "Modeling Daily Arrivals to a Telephone Call center". Management Science, 50, 7, 896-908.

Avramidis, A. N. and P. L'Ecuyer. 2005. "Modeling and Simulation of Call Centers". In Proceedings of the 2005 Winter Simulation Conference, edited by M. E. Kuhl, N. M. Steiger, F. B. Armstrong, and J. A. Joines, 144-152. Piscataway, New Jersey: Institute of Electrical and Electronics Engineers, Inc.

Barlas, P. and C. Heavey. 2016. "KE Tool: An Open Source Software For Automated Input Data In Discrete Event Simulation Projects". In Proceedings of the 2016 Winter Simulation Conference, edited by T. M. K. Roeder, P. I. Frazier, R. Szechtman, E. Zhou, T. Huschka, and S. E. Chick, 472483. Piscataway, New Jersey: Institute of Electrical and Electronics Engineers, Inc.

Barton, R. R. 2012. "Tutorial: Input Uncertainty in Output Analysis". In Proceedings of the 2012 Winter Simulation Conference, edited by C. Laroque, J. Himmelspach, R. Pasupathy, O. Rose, and A. M. Uhrmacher, 67-78. Piscataway, New Jersey: Institute of Electrical and Electronics Engineers, Inc.

Barton, R. R. and L. W. Schruben. 1993. "Uniform and Bootstrap Resampling of Empirical Distributions". In Proceedings of the 1993 Winter Simulation Conference, edited by G.W. Evans, M. Mollaghasemi, E.C. Russell and W.E. Biles, 503-508. Piscataway, New Jersey: Institute of Electrical and Electronics Engineers, Inc.

Barton, R. R., B. L. Nelson, and W. Xie. (2013). "Quantifying Input Uncertainty via Simulation Confidence Intervals". INFORMS Journal on Computing, Articles in Advance, 1-14.

Bause, F., P. Buchholz, and J. Kriege. 2009. "A Comparison of Markovian Arrival and ARMA/ARTA Processes for the Modeling of Correlated Input Processes". In Proceedings of the 2009 Winter Simulation Conference, edited by M. D. Rossetti, R. R. Hill, B. Johansson, A. Dunkin, and R. G. Ingalls, 634-645. Piscataway, New Jersey: Institute of Electrical and Electronics Engineers, Inc.

Bengtsson, N., G. Shao, B. Johansson, T. Lee, S. Leong, A. Skoogh, and C. McLean. 2009. "Input Data Management Methodology for Discrete Event Simulation". In Proceedings of the 2009 Winter Simulation Conference, edited by M. D. Rossetti, R. R. Hill, B. Johansson, A. Dunkin, and R. G. Ingalls, 1335-1344. Piscataway, New Jersey: Institute of Electrical and Electronics Engineers, Inc.

Biller, B. and C. Gunes. 2010. "Capturing Parameter Uncertainty in Simulations with Correlated Inputs". In Proceedings of the 2010 Winter Simulation Conference, edited by B. Johansson, S. Jain, J. Montoya-Torres, J. Hugan, and E. Yucesan, 1167-1177. Piscataway, New Jersey: Institute of Electrical and Electronics Engineers, Inc.

Biller, B. and B. L. Nelson. 2002. "Answers to the Top Ten Input Modeling Questions". In Proceedings of the 2002 Winter Simulation Conference, edited by E. Yücesan, C.-H. Chen, J. L. Snowdon, and J. M. Charnes, 35-40. Piscataway, New Jersey: Institute of Electrical and Electronics Engineers, Inc.

Cario, M. C. and B. L. Nelson. 1996. "Autoregressive to anything: Time-series Input Processes for Simulation". Operations Research Letters, 19, 2, 51-58.

Channouf, N. and P. L'Ecuyer. 2012. "A Normal Copula Model for the Arrival Process in Call Centers". International Transactions in Operational Research, 19, 771-787. 
Channouf, N. and P. L'Ecuyer. 2009. "Fitting a Normal Copula for a Multivariate Distribution with both Discrete and Continuous Marginals". In Proceedings of the 2009 Winter Simulation Conference, edited by M. D. Rossetti, R. R. Hill, B. Johansson, A. Dunkin, and R. G. Ingalls, 352-358. Piscataway, New Jersey: Institute of Electrical and Electronics Engineers, Inc.

Chavez-Demoulin, V., P. Embrechts, and M. Hofert. 2016. "An Extreme Value Approach for Modeling Operational Risk Losses depending on Covariates". Journal of Risk and Insurance, 83(3), 735-776.

Cheng, R. C. H. 1977. "The Generation of Gamma Variables with Non-Integral Shape Parameters". Journal of the Royal Statistical Society, Series C, 26, 1, 71-75.

Cheng, R. C. H. 1993. "Selecting Input Models and Random Variate Generation". In Proceedings of the 1993 Winter Simulation Conference, edited by G. W. Evans, M. Mollaghasemi, E. C. Russell, and W. E. Biles, 34-40. Piscataway, New Jersey: Institute of Electrical and Electronics Engineers, Inc.

Cheng, R. C. H. 1994. "Selecting Input Models". In Proceedings of the 1994 Winter Simulation Conference, edited by J. D. Tew, S. Manivannan, D. A. Sadowski, and A. F. Seila, 184-191. Piscataway, New Jersey: Institute of Electrical and Electronics Engineers, Inc.

Cheng R. C. H. 2006. "Validating and Comparing Simulation Models Using Resampling". Journal of Simulation, 1, 1, 53-63

Cheng, R. C. H. 2015. "Bootstrap Confidence Bands and Goodness-of-Fit Tests in Simulation Input/Output Modelling”. In Proceedings of the 2015 Winter Simulation Conference, edited by L. Yilmaz, W. K. V. Chan, I. Moon, T. M. K. Roeder, C. Macal, and M. D. Rossetti, 16-30. Piscataway, New Jersey: Institute of Electrical and Electronics Engineers, Inc.

Cheng, R. C. H. 2017. Non-standard Parametric Statistical Inference. Oxford: Oxford University Press.

Cheng, R. C. H. and C. S. M. Currie. 2003. "Prior and Candidate Models in the Bayesian Analysis of Finite Mixtures". In Proceedings of the 2003 Winter Simulation Conference, edited by S. Chick, P. J. Sanchez, D. Ferrin, and D. J. Morrice, 392-398. Piscataway, New Jersey: Institute of Electrical and Electronics Engineers, Inc.

Cheng, R. C. H., B. E. Evans, and J. E. Williams. 1988. "Confidence Band Estimations for Distributions used in Probabilistic Design". Intemational Journal of Mechanical Sciences, $30,835-845$.

Cheng, R. C. H. and W. Holland. 1995. "The Effect of Input Parameters on the Variability of Simulation Output". In Proceedings of the Second United Kingdom Simulation Society Conference, edited by R. C. H. Cheng and R. J. Pooley, 29-36. North Berwick, April 1995: Edinburgh University.

Cheng, R. C. H., and W. Holland. 1997. "Sensitivity of Computer Simulation Experiments to Errors in Input Data". Journal of Statistical Computation and Simulation 57, 219-241.

Cheng, R. C. H., and W. Holland. 1998. "Two-Point Methods for Assessing Variability in Simulation Output". Journal of Statistical Computation and Simulation, 60, 183-205.

Cheng, R. C. H., and W. Holland. 2004. "Calculation of Confidence Intervals for Simulation Output". ACM Transactions on Modeling and Computer Simulation, 14, 344-362.

Chernick, M. R. 2008. Bootstrap Methods, A Practitioner's Guide. 2nd ed. New York: Wiley.

Chick, S. E. 1997. "Bayesian Analysis for Simulation Input and Output". In Proceedings of the 2015 Winter Simulation, edited by S. Andradóttir, K. J. Healy, D. H. Withers, and B. L. Nelson, 253-260. Piscataway, New Jersey: Institute of Electrical and Electronics Engineers, Inc.

Chick, S. E. 2001. "Input Distribution Selection for Simulation Experiments: Accounting for Input Uncertainty". Operations Research, 49, 5, 744-758.

Cooper, N. R., J. Margetson, and S. Humble. 1986. "Probability of Failure Calculations and Confidence Band Estimates for an Annular Brittle Disc under Centrifugal Loading”. Journal of Strain Analysis, $21,121-126$.

Corlu, C. G. and B. Biller. 2015. "Subset Selection for Simulations Accounting for Input Uncertainty". In Proceedings of the 2015 Winter Simulation Conference, edited by L. Yilmaz, W. K. V. Chan, I. 


\section{Cheng}

Moon, T. M. K. Roeder, C. Macal, and M. D. Rossetti, 437-446. Piscataway, New Jersey: Institute of Electrical and Electronics Engineers, Inc.

Devroye, L. 1986. Non-Uniform Random Variate Generation. New York: Springer-Verlag.

Embrechts, P., A. McNeil, and D. Straumann. 2017. "Correlation and Dependence in Risk Management: Properties and Pitfalls". ftp://ftp.sam.math.ethz.ch/pub/risklab/papers/CorrelationPitfalls.pdf Accessed 24 April 2017.

Embrechts, P., B. Wang, and R. Wang. 2015. "Aggregation-Robustness and Uncertainty of Regulatory Risk Measures”. Finance and Stochastics, 19(4), 763-790.

Embrechts, P., M. Hofert, and R. Wang. 2016. "Bernoulli and Tail-dependence Compatibility". Annals of Applied Probability 26, 3, 1636-1658.

Fishman, G. S. 1973. Concepts and Methods in Discrete Event Digital Simulation. New York: Wiley.

Fishman, G. S. 1996. Monte Carlo Concepts, Algorithms, and Applications. New York: Springer.

Fishman, G. S. 2001. Discrete-Event Simulation Modeling, Programming, and Applications. New York: Springer.

Fishman, G. S. 2006. A First Course in Monte Carlo. Belmont, CA: Duxbury.

Freimer, M. and L. Schruben. 2002. "Collecting Data and Estimating Parameters for Input Distributions". In Proceedings of the 2002 Winter Simulation Conference, edited by E. Yücesan, C.-H. Chen, J. L. Snowdon, and J. M. Charnes, 392-399. Piscataway, New Jersey: Institute of Electrical and Electronics Engineers, Inc.

Gupta, A. and E. Parzen. 2004. "Input Modeling using Quantile Statistical Methods". In Proceedings of the 2004 Winter Simulation Conference, edited by R. G. Ingalls, M. D. Rossetti, J. S. Smith, and B. A. Peters, 728-736. Piscataway, New Jersey: Institute of Electrical and Electronics Engineers, Inc.

Hammersley, J. M. and D. C. Handscomb. 1964. Monte Carlo Methods. London: Methuen \& Co. Ltd.

Henclewood, D., W. Suh, M. Rodgers, M. Hunter, and R. Fujimoto. 2012. "A Case for Real-time Calibration of Data-driven Microscopic Traffic Simulation Tools". In Proceedings of the 2012 Winter Simulation Conference, edited by C. Laroque, J. Himmelspach, R. Pasupathy, O. Rose, and A. M. Uhrmacher, 1670-1681. Piscataway, New Jersey: Institute of Electrical and Electronics Engineers, Inc.

Hjorth, U. 1994. Computer Intensive Statistical Methods. London: Chapman \& Hall.

Huang Y., M. D. Seck, and A. Verbraeck. 2011. "From Data to Simulation Models: Component-based Model Generation with a Data-driven Approach". In Proceedings of the 2011 Winter Simulation Conference, edited by S. Jain, R. R. Creasey, J. Himmelspach, K. P. White, and M. Fu, 3724-3734. Piscataway, New Jersey: Institute of Electrical and Electronics Engineers, Inc.

Ibrahim, R., P. L'Ecuyer, N. Régnard, and H. Shen. "On the Modeling and Forecasting of Call Center Arrivals". In Proceedings of the 2012 Winter Simulation Conference, edited by C. Laroque, J. Himmelspach, R. Pasupathy, O. Rose, and A. M. Uhrmacher, 256-267. Piscataway, New Jersey: Institute of Electrical and Electronics Engineers, Inc.

Ibrahim, R., P. L'Ecuyer, H. Shen, and M. Thiongane. 2016. "Inter-dependent, Heterogeneous, and Timevarying Service-time Distributions in Call Centers". European Journal of Operations Research, 250, 480-492.

Iman, R. L. 1986. "Modeling Input Processes". In Proceedings of the 1986 Winter Simulation Conference, edited by J. Wilson, J. Henriksen, and S. Roberts, 34-37. New York: Institute of Electrical and Electronics Engineers, Inc.

Jaoua, A., P. L'Ecuyer, and L. Delorme. 2013. "Call-type Dependence in Multiskill Call Centers". Simulation: Transactions of the Society for Modeling and Simulation International, 89, 6, 722-734.

Johnson, M. E. 1987. "Modeling and Generating Input Processes". In Proceedings of the 1987 Winter Simulation Conference, edited by A.Thesen, H. Grant, and W. D. Kelton, 31-32. New York: Institute of Electrical and Electronics Engineers, Inc. 


\section{Cheng}

Kelton, W. D. 1984. "Input Data Collection and Analysis". In Proceedings of the 1984 Winter Simulation Conference, edited by S. Sheppard, U. Pooch, and D. Pegden, 91-95. Piscataway, New Jersey: Institute of Electrical and Electronics Engineers, Inc.

Kelton, W. D. 2007. “Representing and Generating Uncertainty Effectively”. In Proceedings of the 2007 Winter Simulation Conference, edited by S. G. Henderson, B. Biller, M.-H. Hsieh, J. Shortle, J. D. Tew, and R. R. Barton, 38-42. Piscataway, New Jersey: Institute of Electrical and Electronics Engineers, Inc.

Kelton, W. D. 2009. "Representing and Generating Uncertainty Effectively". In Proceedings of the 2009 Winter Simulation Conference, edited by M. D. Rossetti, R. R. Hill, B. Johansson, A. Dunkin, and R. G. Ingalls, 40-44. Piscataway, New Jersey: Institute of Electrical and Electronics Engineers, Inc.

Kendall, D. G. 1974. "Pole Seeking Brownian Motion and Bird Navigation." Journal of the Royal Statistical Society, Series B, 36, 365-417.

Konrad, R. and M. Lawley. 2009. "Input Modeling for Hospital Simulation Models Using Electronic Messages". In Proceedings of the 2009 Winter Simulation Conference, edited by M. D. Rossetti, R. R. Hill, B. Johansson, A. Dunkin and R. G. Ingalls, 134-147. Piscataway, New Jersey: Institute of Electrical and Electronics Engineers, Inc.

Kotiadis, K. 2016. "Towards Self-adaptive Discrete Event Simulation (SADES)". In Proceedings of the Operational Research Society Simulation Workshop 2016 (SW16), edited by A. Anagnostou, K. Hoad, and M. Kunc, 181-191. Birmingham UK: OR Society.

Kuhl, M. E., H. Damerdji, and J. R. Wilson. 1998. "Least Squares Estimation of Nonhomogeneous Poisson Processes". In Proceedings of the 1998 Winter Simulation Conference, edited by D.J. Medeiros, E.F. Watson, J.S. Carson, and M.S. Manivannan, 637-645. Piscataway, New Jersey: Institute of Electrical and Electronics Engineers, Inc.

Kuhl, M. E., E. K. Lada, N. M. Steiger, M. A. Wagner, and J. R. Wilson. 2008. "Introduction to Modeling and Generating Probabilistic Input Processes for Simulation". In Proceedings of the 2008 Winter Simulation Conference, edited by S. J. Mason, R. R. Hill, L. Mönch, O. Rose, T. Jefferson, and J. W. Fowler, 48-61. Piscataway, New Jersey: Institute of Electrical and Electronics Engineers, Inc.

Kuhl, M. E., S. G. Sumant, and J. R. Wilson. 2003. "A Flexible Automated Procedure for Modeling Complex Arrival Processes". In Proceedings of the 2003 Winter Simulation Conference, edited by S. Chick, P. J. Sanchez, D. Ferrin, and D. J. Morrice, 399-407. Piscataway, New Jersey: Institute of Electrical and Electronics Engineers, Inc.

Kurowicka, D. and R. M. Cooke. 2002. "The Vine Copula Method for Representing High Dimensional Dependent Distributions: Application to Continuous Belief Nets". In Proceedings of the 2002. Winter Simulation Conference, edited by E. Yücesan, C.-H. Chen, J. L. Snowdon, and J. M. Charnes, 270278. Piscataway, New Jersey: Institute of Electrical and Electronics Engineers, Inc.

Lada, E. K., N. M. Steiger, M. A. Wagner, and J. R. Wilson. 2005. "Introduction to Modeling and Generating Probabilistic Input Processes for Simulation". In Proceedings of the 2005 Winter Simulation Conference edited by M. E. Kuhl, N. M. Steiger, F. B. Armstrong, and J. A. Joines, 41-55. Piscataway, New Jersey: Institute of Electrical and Electronics Engineers, Inc.

Law, A. M. 2011. "How to Select Simulation Input Probability Distributions". In Proceedings of the 2011 Winter Simulation Conference, edited by S. Jain, R. R. Creasey, J. Himmelspach, K. P. White, and M. $\mathrm{Fu}, 1394-1407$. Piscataway, New Jersey: Institute of Electrical and Electronics Engineers, Inc.

Law, A. M. 2015. Simulation Modeling \& Analysis. $5^{\text {th }}$ ed. New York: McGraw-Hill Education.

Law, A. M. 2016. "A Tutorial on How to Select Simulation Input Probability Distributions". In Proceedings of the 2016 Winter Simulation Conference, edited by T. M. K. Roeder, P. I. Frazier, R. Szechtman, E. Zhou, T. Huschka, and S. E. Chick, 103-117. Piscataway, New Jersey: Institute of Electrical and Electronics Engineers, Inc.

Law, A. M. and S. G. Vincent. 1988. "A Tutorial on UniFit: A System for Total Support of Simulation Input Modeling". In Proceedings of the 1988 Winter Simulation Conference, edited by M. Abrams, P. 


\section{Cheng}

Haigh, and J. Comfort, 188-193. Piscataway, New Jersey: Institute of Electrical and Electronics Engineers, Inc.

Leemis, L. M. 1995. "Input Modeling”. In Proceedings of the 1994 Winter Simulation Conference, edited by J. D. Tew, S. Manivannan, D. A. Sadowski, and A. F. Seila, 55-61. Piscataway, New Jersey: Institute of Electrical and Electronics Engineers, Inc.

Leemis, L. M. 1997. "Seven Habits of Highly Successful Input Modelers". In Proceedings of the 1997 Winter Simulation Conference, edited by S. Andradóttir, K. J. Healy, D. H. Withers, and B. L. Nelson, 39-46. Piscataway, New Jersey: Institute of Electrical and Electronics Engineers, Inc.

Leemis, L. M. 2004. "Building Credible Input Models". In Proceedings of the 2004 Winter Simulation Conference, edited by R. G. Ingalls, M. D. Rossetti, J. S. Smith, and B. A. Peters, 29-40. Piscataway, New Jersey: Institute of Electrical and Electronics Engineers, Inc.

Lin, Y., E. Song, and B. L. Nelson. 2015. "Single Experiment Input Uncertainty". Journal of Simulation, 9, 3, 249-259.

Matus, O., J. Barrera, E. Moreno, and G. Rubino. 2016. "Calibrating a Dependent Failure Model for Computing Reliabilities in Telecommunication Networks". In Proceedings of the 2016 Winter Simulation Conference, edited by T. M. K. Roeder, P. I. Frazier, R. Szechtman, E. Zhou, T. Huschka, and S. E. Chick, 490-500. Piscataway, New Jersey: Institute of Electrical and Electronics Engineers.

Morgan, L. E., A. C. Titman, D. J. Worthington, and B. L. Nelson. 2016. "Input Uncertainty Quantification for Simulation Models with Piecewise-constant Non-stationary Poisson Arrival Processes". In Proceedings of the 2016 Winter Simulation Conference, edited by T. M. K. Roeder, P. I. Frazier, R. Szechtman, E. Zhou, T. Huschka, and S. E. Chick, 370-381. Piscataway, New Jersey: Institute of Electrical and Electronics Engineers, Inc.

Nagaraj, K. and R. Pasupathy. 2015. "Modeling Dependence in Simulation Input: the Case for Copulas". In Proceedings of the 2015 Winter Simulation Conference, edited by L. Yilmaz, W. K. V. Chan, I. Moon, T. M. K. Roeder, C. Macal, and M. D. Rossetti, 1850-1864. Piscataway, New Jersey: Institute of Electrical and Electronics Engineers, Inc.

Neuts, M. F. and M. E. Pagano. 1981. "Generating Random Variates from a Distribution of Phase Type". In Proceedings of the 1981 Winter Simulation Conference, edited by T.I. Oren, C. M. Delfosse, and C.M. Shub, 381-387. Piscataway, New Jersey: Institute of Electrical and Electronics Engineers, Inc.

Oreshkin, B. N., N. Régnard, and P. L'Ecuyer. 2016. "Rate-Based Daily Arrival Process Models with Application to Call Centers". Operations Research, 64, 2, 510-525.

Rabe, M. and A. A. Scheidler. 2014. "An Approach for Increasing the Level of Accuracy in Supply Chain Simulation by Using Patterns on Input Data". In Proceedings of the 2014 Winter Simulation Conference, edited by A. Tolk, S. Y. Diallo, I. O. Ryzhov, L. Yilmaz, S. Buckley, and J. A. Miller, 1897-1906. Piscataway, New Jersey: Institute of Electrical and Electronics Engineers, Inc.

Richardson, S. and P. Green. 1997. "On Bayesian Analysis of Mixtures with an Unknown Number of Components". J. Roy, Statist. Soc. B, 59, 731-792.

Schmeiser, B. W. 1977. "Methods for Modelling and Generating Probabilistic Components in Digital Computer Simulation when the Standard Distributions are not Adequate: A Survey". In Proceedings of the Winter Simulation Conference, National Bureau of Standards, Gaithersburg, MD, December 1977, 50-57.

Shanker, A. and W. D. Kelton. 1991. "Empirical Input Distributions: An Alternative to Standard Input Distributions in Simulation Modeling". In Proceedings of the 1991 Winter Simulation Conference, edited by B. L. Nelson, W. D. Kelton, and G. M. Clark, 978-985. Piscataway, New Jersey: Institute of Electrical and Electronics Engineers, Inc.

Skoogh, A. and B. Johansson. 2008. "A Methodology for Input Data Management in Discrete Event Simulation Projects". In Proceedings of the 2008 Winter Simulation Conference, edited by S. J. Mason, R. R. Hill, L. Mönch, O. Rose, T. Jefferson, and J. W. Fowler, 1727-1735. Piscataway, New Jersey: Institute of Electrical and Electronics Engineers, Inc. 


\section{Cheng}

Song, E. and B. L. Nelson. 2013. "A Quicker Assessment of Input Uncertainty”. In Proceedings of the 2013 Winter Simulation Conference, edited by R. Pasupathy, S.-H. Kim, A. Tolk, R. Hill, and M. E. Kuhl, 474-485. Piscataway, New Jersey: Institute of Electrical and Electronics Engineers, Inc.

Song, E., B. L. Nelson, and D. Pegden. 2014. "Advanced Tutorial: Input Uncertainty Quantification”. In Proceedings of the 2014 Winter Simulation Conference, edited by A. Tolk, S. Y. Diallo, I. O. Ryzhov, L. Yilmaz, S. Buckley, and J. A. Miller, 162-176. Piscataway, New Jersey: Institute of Electrical and Electronics Engineers, Inc.

Song, E. and B. L. Nelson. 2015. "Quickly Assessing Contributions to Input Uncertainty". IIE Transactions 47, 893-909.

Steckley, S. G. and S. G. Henderson. 2003. "A Kernel Approach to Estimating the Density of a Conditional Distribution". In Proceedings of the 2003 Winter Simulation Conference, edited by S. Chick, P. J. Sanchez, D. Ferrin, and D. J. Morrice, 383-391. Piscataway, New Jersey: Institute of Electrical and Electronics Engineers, Inc.

Stephens, M. A. 1974. "EDF Statistics for Goodness of Fit and some Comparisons". J. Amer. Statist. Assoc. 69, 730-737.

Tannock, J., B. Cao, R. Farr, and M. Byrn. 2007. "Data-driven Simulation of the Supply Chain - Insights from the Aerospace Sector". Int. J. Production Economics, 110, 70-84.

Tocher, K. D. 1963. The Art of Simulation. London: Hodder and Stoughton.

Uhlig, T., S. Rank, and O. Rose. 2016, "Evaluation of Modeling Tools for Autocorrelated Input Processes". In Proceedings of the 2016 Winter Simulation Conference, edited by T. M. K. Roeder, P. I. Frazier, R. Szechtman, E. Zhou, T. Huschka, and S. E. Chick, 1048-1059. Piscataway, New Jersey: Institute of Electrical and Electronics Engineers, Inc.

Vincent, S. G. and A. M. Law. 1990. "A Tutorial on UniFit II: A System for Total Support of Simulation Input Modeling". In Proceedings of the 1990 Winter Simulation Conference, edited by O. Balci, R. P. Sadowski, and R. E. Nance, 180-183. Piscataway, New Jersey: Institute of Electrical and Electronics Engineers, Inc.

Wilson, J. R. 1997. "Modeling Dependencies in Stochastic Simulation Inputs". In Proceedings of the 1997 Winter Simulation Conference, edited by S. Andradóttir, K. J. Healy, D. H. Withers, and B. L. Nelson, 47-52. Piscataway, New Jersey: Institute of Electrical and Electronics Engineers, Inc.

Xie, W., B. L. Nelson, and R. R. Barton. 2014a. "Statistical Uncertainty Analysis for Stochastic Simulation with Dependent Input Models". In Proceedings of the 2014 Winter Simulation Conference, edited by A. Tolk, S. Y. Diallo, I. O. Ryzhov, L. Yilmaz, S. Buckley, and J. A. Miller, 674-685. Piscataway, New Jersey: Institute of Electrical and Electronics Engineers, Inc.

Xie, W., B. L. Nelson, and R. R. Barton. 2014b. "A Bayesian Framework for Quantifying Uncertainty in Stochastic Simulation”. Operations Research, 62, 6, 1439-1452.

Xie, W., B. L. Nelson, and R. R. Barton. 2016. "Multivariate Input Uncertainty in Output Analysis for Stochastic Simulation". ACM Trans. Model. Comput. Simul. 27, 1, Article 5, 22 pages.

Zouaoui, F., and J. R. Wilson. 2001. "Accounting for Parameter Uncertainty in Simulation Input Modeling". In Proceedings of the 2001 Winter Simulation Conference, edited by B. A. Peters, J. S. Smith, M. W. Rohrer, and D. J. Medeiros, 290-299. Piscataway, New Jersey: Institute of Electrical and Electronics Engineers, Inc.

Zouaoui, F., and J. R. Wilson. 2003. "Accounting for Parameter Uncertainty in Simulation Input Modeling". IIE Transactions 35, 3, 781-792.

Zouaoui, F., and J. R. Wilson. 2004. "Accounting for Input-model and Input-parameter Uncertainties in Simulation". IIE Transactions 36 ,11, 1135-1151. 


\section{AUTHOR BIOGRAPHY}

RUSSELL CHENG is Emeritus Professor of Operational Research at the University of Southampton. He has an M.A. and the Diploma in Mathematical Statistics from Cambridge University, England. He obtained his Ph.D. from Bath University. He is a former Chairman of the U.K. Simulation Society, a former Fellow of the Royal Statistical Society and a Fellow of the Institute of Mathematics and Its Applications. His research interests include: design and analysis of simulation experiments and parametric estimation methods. He was a Joint Founding Editor of the IMA Journal of Management Mathematics. He was awarded the INFORMS Simulation Society Lifetime Professional Achievement Award in 2016. His email address is cheng@btinternet.com. 